\title{
Long Term Outcomes of Cataract Surgery in Severe and End Stage Glaucoma with Controlled IOP: A Retrospective Study
}

\section{Lin $\mathrm{Fu}$}

Wenzhou Medical University

\section{Yau Kei Chan}

University of Hong Kong

Junhua Li

Wenzhou Medical University

Li Nie

Wenzhou Medical University

$\mathrm{Na} \mathrm{Li}$

Wenzhou Medical University

Weihua Pan ( $\triangle$ pan_weihua@163.com )

Affiliated Eye Hospital, Wenzhou Medical University https://orcid.org/0000-0003-3975-4806

\section{Research article}

Keywords: cataract extraction, severe and end stage of glaucoma, visual acuity, intraocular pressure

Posted Date: November 22nd, 2019

DOI: https://doi.org/10.21203/rs.2.17700/v1

License: (1) This work is licensed under a Creative Commons Attribution 4.0 International License. Read Full License 


\section{Abstract}

Background: To investigate the long term surgical outcomes of cataract surgery in severe and end glaucoma patients with preoperative intraocular pressure less than $21 \mathrm{mmHg}$, and to detect the associated factors.

Methods: A retrospective study of severe and end stage glaucoma patients who underwent cataract surgery or combined with goniosynechialysis from March 2015 to April 2018. Main outcome measures were visual acuity, intraocular pressure, number of glaucoma medications and complications.

Results: Twenty patients (24 eyes) were included. The mean age was $64.6 \pm 11.0$ years and the mean followed up duration was $21.4 \pm 7.3$ months. The final visual acuity was significantly improved from $0.93 \pm 0.72$ to $0.70 \pm 0.74$, within 14 (58.3\%) eyes improved, 5 (20.8\%) eyes kept unchanged, and $5(20.8 \%)$ eyes reduced. Linear regression analysis indicated that higher mean deviation, higher visual field index and lower glaucoma stage associated with greater final visual acuity improvement $(r=-0.545, r=-0.501, r$ $=0.521$ respectively). Moreover, the final number of medications were reduced from $1.1 \pm 0.9$ to $0.2 \pm 0.5$ $(p<0.01)$. The mean intraocular pressure was not significantly reduced with the final IOP of $13.2 \pm 3.9$ $(6.8-25.9) \mathrm{mmHg}(p=0.97)$. While the eyes with intraocular pressure above $15 \mathrm{mmHg}$ was reduced to 7 $(29.2 \%)$ eyes compared to $11(45.8 \%)$ eyes at baseline. Moreover, the number of eyes without the use of medications was increased from $6(25 \%)$ preoperatively to 20 (83.3\%) eyes postoperatively.

Conclusions: Final visual acuity was significantly improved in the severe and end stage glaucoma patients and the number of eyes came off medications increased by $58.3 \%$ after cataract surgery. Preoperatively, the glaucoma stage, mean deviation and visual field index are important parameters to predict the visual acuity changes after cataract surgery.

\section{Introduction}

Glaucoma and cataract constitute the top two leading causes of blindness worldwide which significantly affect the visual impairment and quality of life $[1,2]$. They are commonly coexisted as both are agerelated $[3,4]$. Their relative impact on the visual function are difficult to differentiate when they are coexisting. Meanwhile, cataract can have a great influence on quality of life in glaucoma patients with differed severities [5].

However, in severe and end stage glaucoma, postoperative "wipe out" is a risky complication. The "wipe out" is defined as a sudden visual acuity (VA) loss without apparent reasons especially in advanced glaucoma after filtering surgery [6, 7]. Moreover, eyes with glaucoma are at increased risk of complications like posterior capsular tear with vitrectomy, postoperative inflammation, prolonged increase of intraocular pressure (IOP) and achieve less significant visual improvement than eyes without glaucoma after cataract surgery [8]. Accordingly, only patients with medically uncontrolled glaucoma may warrant a surgery. Conservative managements, instead of cataract surgery, are much preferred for glaucoma patients with controlled IOP. 
Glaucomatous eyes, as reported, can still gain satisfactory visual outcomes after phacoemulsification [8]. Combined phacoemulsification and non-penetrating deep sclerectomy (NPDS) was ever performed in the severe and end stage glaucoma patients, no "wipe out" was observed and the mean VA was improved at month -6 postoperatively [9]. Another study demonstrated that $73 \%$ glaucoma patients with low vision had better vision or maintained at 5 years after cataract surgery [10]. The improved visual function like VA and visual field index, is suggested to be related with better quality of life in glaucoma patients [11]. Therefore, it is advocated that cataract surgery should be offered in glaucoma patients who are IOP controlled. Cataract extraction not only improves the visual function, but also reduces the IOP $[12,13]$. Due to the preservatives in glaucoma medications like benzalkonium chloride (BAK) and sodium perborate, longer treatment period and the use of multiple glaucoma drugs, ocular surface diseases (OSD) are more common in glaucomatous eyes using topical medications than glaucomatous eyes without medications and also normal eyes [14-16]. The IOP lowering effect of cataract surgery can reduce the usage of glaucoma medications, thereby alleviate the OSD and improve patient quality of life. Hence, despite the potential risky complications, cataract surgery is still recommended for severe and end stage glaucoma patients with IOP controlled to improve their life quality.

In previous studies, patients who were defined as medically uncontrolled were with IOP higher than 21 $\mathrm{mmHg}$ or with the use of more than 3 glaucoma medications $[17,18]$. Here, the controlled IOP was defined as IOP lower than $21 \mathrm{mmHg}$, and requiring not more than 3 topical glaucoma drugs. To provide better predication on the VA outcome in severe and end stage glaucoma with controlled IOP, in this work, we retrospectively studied the outcomes of cataract surgery of these patients. The long-term VA outcome, as well as the predictive factors of VA changes in these patients, are studied and reported here.

\section{Materials And Methods}

A retrospective study was conducted to review the medical charts from the high risk surgery bank in our hospital. The severe and end stage glaucoma patients who underwent cataract surgery from March 2015 to April 2018 were retrieved. Patients with a follow-up of at least 1 year and preoperative IOP less than $21 \mathrm{mmHg}$ were included. The investigational study was approved by the Institutional Review Board of the Wenzhou Medical University and in accordance with the tenets of the Declaration of Helsinki.

Referred to the modified Bascom Palmer Glaucoma Staging System, severe glaucoma (stage 4) was confirmed when a mean deviation $(\mathrm{MD})<-20 \mathrm{~dB}$ and one of the following three criteria was met by the 30-2 Humphrey perimetry preoperatively: 1 . on pattern deviation plot, $50 \%$ to $75 \%$ points depressed below the $5 \%$ level or $25 \%$ to $50 \%$ points depressed below the $1 \%$ level; 2 . there were more than 1 points with sensitivity of $0 \mathrm{~dB}$ in the central $5^{\circ}$ area; 3 . at least one point with sensitivity of less than $15 \mathrm{~dB}$ in both hemifields within $5^{\circ}$ of fixation. The end stage glaucoma (stage 5) was defined by the VA $<20 / 200$ or unavailable to perform the Humphrey visual field examination attributable to glaucoma [19]. For the purpose of statistical analysis, the MD of stage 5 eyes were considered as $-33 \mathrm{~dB}$ and the visual field index (VFI) of these eyes were $0 \%$. 
Preoperatively, the following information was collected for each eye: age, gender, glaucoma type, vertical cup to disc ratio, visual field results, IOP, number of glaucoma medication, VA, best corrected visual acuity (BCVA), and surgical methods. After surgery, the VA, IOP, glaucoma medication at the first month, 6th month and final visit, complication and intervention was recorded. The case of study was considered as "wipe out" if the postoperative VA reduced to $<20 / 200$, or to counting fingers or less when preoperative VA was $<20 / 200$. The VA and BCVA was measured as decimal units and converted into a logarithm of the minimum angle of resolution (logMAR). For the purpose of statistical analysis, counting fingers and hand motion were equating to $1 / 200$ and $0.5 / 200$ [20].

Phacoemulsification and intraocular lens implantation (Phaco-IOL) was carried out in these eyes. In angle closure glaucoma (ACG) patients, if peripheral anterior synechia (PAS) was observed under gonioscope in the preoperative examination, combined Phaco-IOL and goniosynechialysis (Phaco-IOL-GSL) was conducted. All the operations were performed by the same experienced surgeon (WH Pan).

The data were analyzed in IBM SPSS software version 23 (Chicago, IL). The numeric parameters were evaluated by Kolmogorov-Smirnov test for the distribution of normality. Kruskal-Wallis test was used to evaluate the categorical parameters. Friedman or one-way analysis of variance (ANOVA) tests was used to compare the quantitative variables. The factors related to VA changes were analyzed by Pearson or Spearman's correlation. They are presented as mean \pm standard deviations (SD) and range. Statistical significance was set at a $p$ value of less than 0.05 .

\section{Results}

From the high risk surgery bank in our hospital from March 2015 to April 2018, 109 glaucoma patients underwent cataract surgery were reviewed (Figure 1). In total, 24 eyes in 20 patients with severe and end stage glaucoma were included for analysis. Eighty nine patients were excluded for the following ordered reasons: 42 patients had a follow up duration less than 1 year, 26 patients had preoperative IOP higher than $21 \mathrm{mmHg}, 6$ did not have complete set of required data, 4 complicated with retinopathy and lens dislocation, 11 had the glaucoma stage below 4 .

Table 1 summarized the baseline characteristics of the patients. Of the 24 eyes, the mean age was $64.6 \pm$ 11.0 (range, 44-80) years old, 58.3\% were male and right eyes, they followed up for $21.4 \pm 7.3$ (range, 12-39) months, only 3 (12.5\%) eyes were normal tension glaucoma (NTG), and the remaining 21 (87.5\%) eyes were primary angle closure glaucoma (PACG). Except phacoemulsification and cataract extraction, 19 eyes of them were underwent combined GSL. The mean number of preoperative medications were 1.1 \pm 0.9 (range, $0-3$ ) with only $6(25 \%)$ eyes came off medications and the mean IOP was $13.8 \pm 3.3$ (range, 8.1-17.9) $\mathrm{mmHg}$. According to the modified Bascom Palmer Glaucoma Staging System, 19 (79.2\%) and $5(20.8 \%)$ of the eyes them were stage 4 and 5 respectively. The mean MD was $-29.16 \pm 4.20 \mathrm{~dB}$ (range, -21.4 to -35 \and the mean VFI was $13.38 \% \pm 11.46 \%$ (range, $0 \%-37 \%$ ).

Changes in baseline and postoperative VA are shown in Figure 2. The mean VA at baseline was $0.93 \pm$ 0.72 (range, 0.1 to 2.6 ) logMAR unit. The VA levels were significantly improved in all the postoperative 
visits at 1 month, 6 months and the final visit $(p<0.01, p<0.01$ and $p<0.05$, Friedman test). They were $0.58 \pm 0.52$ (range, 0.05 to 2.3), $0.63 \pm 0.66$ (range, 0.05 to 2.6) and $0.70 \pm 0.74$ (range, 0.1 to 2.6) logMAR unit respectively. The percentage of postoperative VA improvement, unchanged and reduction are listed in table 2. Final VA improved in $14(58.3 \%)$ eyes and was unchanged in $5(20.8 \%)$ eyes (Table 2$)$. Moreover, no cases of "wipe out" was detected. Linear regression was performed to analyze the factors associated with the VA changes between baseline VA and the final VA. The MD, VFI and glaucoma stage were found to be related to the VA changes ( $r=-0.545, p=0.006 ; r=-0.501, p=0.013 ; r=0.521, p=0.009$ ) (Figure $3)$. These indicated that higher MD, higher VFI and lower glaucoma stage indicated greater final VA improvement.

The final number of antiglaucoma medications were significantly reduced from $1.1 \pm 0.9$ (range, $0-3)$ to $0.2 \pm 0.5$ (range, $0-2)(p<0.01)$. The number of eyes that came off medications increased from preoperatively $6(25 \%)$ eyes to $20(83.3 \%)$ eyes postoperatively (Figure 4$)$. The number of eyes with IOP above $15 \mathrm{mmHg}$ at baseline were $11(45.8 \%)$ and was reduced to $7(29.2 \%)$ at the final visit, although the mean IOP between the baseline and final was not statistically different with the final IOP of $13.2 \pm 3.9$ $(6.8-25.9) \mathrm{mmHg}(p=0.97)$.

The postoperative complications are shown in Table 3. The incidence of posterior capsular opacity (PCO) was $21.4 \%$ and was only observed in the VA improved group within 3 eyes. The time of the PCO detected was 31 months, 12 months and 12 months after surgery in these 3 eyes respectively. All of them were underwent Neodymium: YAG laser posterior capsulotomy immediately. Shallow anterior chamber was found in 1 eye at 12 months after surgery in the VA unchanged group and the laser peripheral iridotomy was performed. Malignant glaucoma occurred in 1 eye of the VA unchanged group 5 days after operation. Anterior vitrectomy was performed and effectively managed this complication. One eye in the VA worsening group experienced ischemic optic neuropathy 10 days postoperatively. Strategies were immediately carried out to rescue the vision, including injection of compound anisodine hydrobromide and oxygen inhalation The final vision of this patient was $1.22 \log M A R$ with baseline VA of $0.92 \log M A R$ unit.

\section{Discussion}

The information of the long term surgical outcomes in severe and end stage glaucoma with controlled IOP is scarce. Particularly, the understanding on the visual outcome of these patients after cataract surgery is lacking. The reported surgical outcomes of cataract surgery in severe and end stage glaucoma in literature were mainly come up from patients who were medically uncontrolled [9, 21]. In glaucoma patients with controlled IOP, cataract surgery is seldomly performed since they are at high risk of "wipe out" [6, 7]. Here, our present retrospective study instead shows that the postoperative VA of these patients significantly improved after cataract surgery. In addition, the number of glaucoma medications also significantly reduced. Also, the baseline MD, VFI and glaucoma stage may help to predict the visual outcome after surgery. 
Cataract extraction was reported to improve the VA in glaucoma patients with most of them were in the early stage or medically uncontrolled. For instance, in the Collaborative Initial Glaucoma Treatment Study (CIGTS), the VA was improved abruptly after cataract extraction and maintained for 1.5 years in glaucoma with preoperative mean deviation of $-5.74 \mathrm{~dB}$ [22]. And in 2018, Igor et al. reported that the VA of severe and end stage glaucoma patients was not improved when combined the glaucoma surgery of NPDS with phacoemulsification [23]. While in his later study, the VA was improved after the same surgeries [9]. Both of the studies were last for 6 months. The difference in conclusion may be due to the difference in sample sizes, with only 5 in the earlier one study and then increased to 18 eyes for the later. Even in these medically uncontrolled eyes, VA can be improved after cataract surgery although the primary purpose was to reduce IOP. We hence speculated that cataract extraction can also improve VA in IOP controlled eyes after surgery in these severe and end stage glaucoma. In clinical settings, IOP is always the primary focus in glaucoma management while VA is rarely considered as an assessment parameter of treatment outcome in glaucoma patients. However, VA is highly reflecting the life quality of patients. For glaucoma patients in the severe and end stage without constricted visual field, VA indeed reflects more about the subject perception and the ability to interact with environment [10]. Accordingly, VA improvement in these patients offer an important opportunity to improve their quality of life. Here, we pioneeringly provided the information that the mean VA improved from $0.93 \pm 0.72$ to $0.70 \pm 0.74 \log$ MAR unit with a mean follow up of $21.4 \pm 7.3$ months, in IOP controlled severe and end stage glaucoma patients. This result is encouraging and useful since little is known about the visual outcome of severe and end stage glaucoma patients with IOP controlled before. It is difficult to quantify the vision reduction contributed by cataract or glaucoma independently but based on this study we can now suggest VA can be improved in these patients after cataract surgery.

The reason that cataract surgery was seldom performed solely for the purpose of visual improvement in severe and end stage glaucoma patients with controlled IOP, is due to the risk of "wipe out". It is a longstanding debate whether cataract surgery should be performed on patients with severe and end stage glaucoma. In the past, the reported incidence of "wipe out" in end stage glaucoma was discrepant. Some suggest this to be a rare or even non-existent complication and others fear the risk of sudden visual loss $[6,21,24]$. In our study, no cases of "wipe out" occurred. Most of the studies that reported high rate of "wipe out" were more than 26 years ago. Nowadays, with the advanced technologies, complications can be well resolved and "wipe out" might have a chance to be relegated to a place in history [25]. "Wipe out" was regarded as a sudden vision loss without apparent causes especially in advanced glaucoma after filtering surgery [6, 7], and was suspected to be related with ocular hypotony during surgery. Although cataract extraction is not a filtering surgery, ocular hypotony may still happen theoretically. In this study, all the surgeries were performed by a single experienced glaucoma specialist, no cases of "wipe out" was observed and $79.1 \%$ eyes showed better or maintained VA at the final visit. These results therefore supported that cataract surgery in general is safe and effective on patients with severe and end stage glaucoma patients. For the eyes with postoperative complications, 3 eyes had PCO and were managed by posterior capsulectomy and their final VA were improved. Malignant glaucoma occurred in one eye and shallow anterior chamber happened in another eye. Both showed unchanged VA in the final checking. The 
only one eye which suffered ischemic optic neuropathy displayed worsening VA. This eye was at stage 5 with poor vision preoperatively. It is not conclusive to determine if the ischemic optic neuropathy or stage 5 contributed to the worsening VA outcome in this case. Nevertheless, from our linear regression analysis, the greater baseline MD, higher VFI and lower glaucoma stage may predict greater VA improvement after cataract surgery. However, VA is not directly related to the visual field. The functional visual acuity (FVA) measured by an AS-28 FVA measurement system had shown a weak correlation with MD in glaucoma with different severities [26]. Here, we hypothesize that the VA may be more associated with visual filed parameters in severely damaged glaucoma. To our knowledge, the visual field parameters and glaucoma stage have not been indicated as the predictive factors of the VA outcomes in severe and end stage glaucoma after cataract surgery. These factors may provide an important reference to the decision management of treatments for these patients.

Cataract extraction was reported to significantly lower IOP in angle closure glaucoma eyes due to the anatomical changes of the narrow anterior segment $[27,28]$. In this study, $87.5 \%$ eyes are cases of ACG. However, in current study the mean IOP was not significantly changed after cataract surgery, from $13.8 \pm$ $3.3 \mathrm{mmHg}$ preoperatively to $13.2 \pm 3.9 \mathrm{mmHg}$ postoperatively. This can be explained that the IOP of these included patients were all in a normal range of IOP (below $21 \mathrm{mmHg}$ ). Nevertheless, it can be noticed that percentage of eyes with IOP above $15 \mathrm{mmHg}$ reduced from $45.8 \%$ at baseline to $29.2 \%$ at the final visit. And it can also be revealed on the other hand, the IOP control after cataract extraction was demonstrated by the decrease of topical glaucoma drugs used in the postoperative period. The number of glaucoma medications were significantly reduced to the mean of $0.2 \pm 0.5$ from the baseline number of $1.1 \pm 0.9$. Also, the percentage of patients who came off topical glaucoma drugs was greatly increased from $25 \%$ to $83.3 \%$. It would be meaningful to investigate how such reduction of the drugs impacts on the quality of life of patients, financial costs and adverse effect from the drugs.

Since this is a retrospective study, we mainly used the VA as the indicator of the success of cataract surgery. Other measures like subjective visual function, color perception and overall satisfaction to surgery were not documented. They may also be important parameters to evaluate the quality of life of patients. Also, due to the limited sample size and follow up duration, the current dataset is insufficient to analyze the significance of glaucomatous progression and related complications. A larger sample size of prospective study is needed for further justifications.

In conclusion, cataract extraction provides an additional opportunity of VA improvement in severe and end stage glaucoma patients with controlled IOP. It may also relief the life burden of patients by reducing or even coming off their topical medications. The prediction of VA outcome can refer to the preoperative visual field parameters including MD, VFI and glaucoma stage. The results from our study may change the traditional management practice of severe and end stage glaucoma with controlled IOP and greatly improve the quality of life of patients.

\section{List Of Abbreviations}


VA visual acuity

IOP intraocular pressure

NPDS non-penetrating deep sclerectomy

BAK benzalkonium chloride

OSD ocular surface diseases

MD mean deviation

VFI visual field index

BCVA best corrected visual acuity

logMAR logarithm of the minimum angle of resolution

Phaco-IOL Phacoemulsification and intraocular lens implantation

ACG angle closure glaucoma

PAS peripheral anterior synechia

GSL goniosynechialysis

ANOVA one-way analysis of variance

SD standard deviations

NTG normal tension glaucoma

PACG primary angle closure glaucoma

PCO posterior capsular opacity

CIGTS Collaborative Initial Glaucoma Treatment Study

FVA functional visual acuity

\section{Declarations}

Ethics approval and consent to participate This study is approved by the institutional review board of the affiliated Eye Hospital of Wenzhou Medical University (Number: 2019-185-K-167). The written informed consents were achieved from all patients. Consent for publication Not applicable. Availability of data and material The datasets used and/or analyzed during the current study are available from the corresponding author on reasonable request. Competing interests The authors declare that they have no 
competing interests. Funding This study was Supported by the Nature and Science Foundation of Zhejiang Province, China (Grant No. LQ19H120002), and also the Wenzhou Municipal Science and Technology Bureau (Grant No. Y20190170), China. Authors' contributions LF, WP were responsible for the design of the study and interpretation of the results. LN, JL and NL conducted the study and collected the data. NL were responsible for the operations. LF and YC analyzed the data. LF was a major contributor in writing the manuscript. LF, WP, YC, LN and JL reviewed the article. All authors read and approved the final manuscript. Acknowledgements The authors are grateful to Hengli Lian for her statistical advice on this manuscript.

\section{References}

1.Guedes RA, Guedes VM, Freitas SM, Chaoubah A: Quality of life of medically versus surgically treated glaucoma patients. J Glaucoma 2013, 22(5):369-373.

2.McKean-Cowdin R, Wang Y, Wu J, Azen SP, Varma R, Los Angeles Latino Eye Study G: Impact of visual field loss on health-related quality of life in glaucoma: the Los Angeles Latino Eye Study. Ophthalmology 2008, 115(6):941-948 e941.

3.Quigley HA, Broman AT: The number of people with glaucoma worldwide in 2010 and 2020. Br J Ophthalmol2006, 90(3):262-267.

4.Congdon N, O'Colmain B, Klaver CC, Klein R, Munoz B, Friedman DS, Kempen J, Taylor HR, Mitchell P, Eye Diseases Prevalence Research G: Causes and prevalence of visual impairment among adults in the United States. Arch Ophthalmol 2004, 122(4):477-485.

5.Skalicky SE, Martin KR, Fenwick E, Crowston JG, Goldberg I, McCluskey P: Cataract and quality of life in patients with glaucoma. Clin Exp Ophthalmol 2015, 43(4):335-341.

6.Costa VP, Smith M, Spaeth GL, Gandham S, Markovitz B: Loss of visual acuity after trabeculectomy. Ophthalmology 1993, 100(5):599-612.

7.Lichter PR, Ravin JG: Risks of sudden visual loss after glaucoma surgery. Am J Ophthalmol 1974, 78(6):1009-1013.

8.Turalba A, Payal AR, Gonzalez-Gonzalez LA, Cakiner-Egilmez T, Chomsky AS, Vollman DE, Baze EF, Lawrence M, Daly MK: Cataract Surgery Outcomes in Glaucomatous Eyes: Results From the Veterans Affairs Ophthalmic Surgery Outcomes Data Project. Am J Ophthalmol 2015, 160(4):693-701 e691.

9.Leleu I, Penaud B, Blumen-Ohana E, Rodallec T, Adam R, Laplace O, Akesbi J, Nordmann JP: Risk assessment of sudden visual loss following non-penetrating deep sclerectomy in severe and end-stage glaucoma. Eye (Lond) 2019, 33(6):902-909. 
10.Monestam El, Lundqvist BM, Jonsson AC: Long-time visual functional results of cataract surgery on low vision patients. Clin Ophthalmol 2008, 2(1):187-194.

11.Xu X, Sun Q, Ma YY, Zou HD: Vision-related Quality of Life Outcomes of Cataract Surgery in Advanced Glaucoma Patients. J Glaucoma 2016, 25(1):e5-11.

12.Melancia D, Abegao Pinto L, Marques-Neves C: Cataract surgery and intraocular pressure. Ophthalmic Res 2015, 53(3):141-148.

13. Coh P, Moghimi S, Chen RI, Hsu CH, Masis Solano M, Porco T, Lin SC: Lens Position Parameters as Predictors of Intraocular Pressure Reduction After Cataract Surgery in Glaucomatous Versus Nonglaucomatous Eyes. Invest Ophthalmol Vis Sci 2016, 57(6):2593-2599.

14.Perez-Bartolome F, Martinez-de-la-Casa JM, Arriola-Villalobos P, Fernandez-Perez C, Polo V, GarciaFeijoo J: Ocular Surface Disease in Patients under Topical Treatment for Glaucoma. Eur J Ophthalmol 2017, 27(6):694-704.

15.Ramli N, Supramaniam G, Samsudin A, Juana A, Zahari M, Choo MM: Ocular Surface Disease in Glaucoma: Effect of Polypharmacy and Preservatives. Optom Vis Sci 2015, 92(9):e222-226.

16.Goldberg I, Graham SL, Crowston JG, d'Mellow G, Australian, New Zealand Glaucoma Interest G: Clinical audit examining the impact of benzalkonium chloride-free anti-glaucoma medications on patients with symptoms of ocular surface disease. Clin Exp Ophthalmol 2015, 43(3):214-220.

17.Tham CC, Kwong YY, Leung DY, Lam SW, Li FC, Chiu TY, Chan JC, Lam DS, Lai JS:

Phacoemulsification versus combined phacotrabeculectomy in medically uncontrolled chronic angle closure glaucoma with cataracts. Ophthalmology 2009, 116(4):725-731, 731 e721-723.

18. Tham CC, Kwong YY, Baig N, Leung DY, Li FC, Lam DS: Phacoemulsification versus trabeculectomy in medically uncontrolled chronic angle-closure glaucoma without cataract. Ophthalmology 2013, 120(1):62-67.

19.Mills RP, Budenz DL, Lee PP, Noecker RJ, Walt JG, Siegartel LR, Evans SJ, Doyle JJ: Categorizing the stage of glaucoma from pre-diagnosis to end-stage disease. Am J Ophthalmol 2006, 141(1):24-30.

20.Gregori NZ, Feuer W, Rosenfeld PJ: Novel method for analyzing snellen visual acuity measurements. Retina 2010, 30(7):1046-1050.

21.Topouzis F, Tranos P, Koskosas A, Pappas T, Anastasopoulos E, Dimitrakos S, Wilson MR: Risk of sudden visual loss following filtration surgery in end-stage glaucoma. Am J Ophthalmol2005, 140(4):661-666.

22.Musch DC, Gillespie BW, Niziol LM, Janz NK, Wren PA, Rockwood EJ, Lichter PR, Collaborative Initial Glaucoma Treatment Study G: Cataract extraction in the collaborative initial glaucoma treatment study: 
incidence, risk factors, and the effect of cataract progression and extraction on clinical and quality-of-life outcomes. Arch Ophthalmol 2006, 124(12):1694-1700.

23.Leleu I, Penaud B, Blumen-Ohana E, Rodallec T, Adam R, Laplace O, Akesbi J, Nordmann JP: Central 10-degree visual field change following non-penetrating deep sclerectomy in severe and end-stage glaucoma: preliminary results. Graefes Arch Clin Exp Ophthalmol 2018, 256(8):1489-1498.

24.Aggarwal SP, Hendeles S: Risk of sudden visual loss following trabeculectomy in advanced primary open-angle glaucoma. Br J Ophthalmol 1986, 70(2):97-99.

25.Moster MR, Moster ML: Wipe-out: a complication of glaucoma surgery or just a blast from the past? Am J Ophthalmol 2005, 140(4):705-706.

26.Ozeki N, Yuki K, Shiba D, Tsubota K: Evaluation of Functional Visual Acuity in Glaucoma Patients. J Glaucoma 2017, 26(3):223-226.

27. Hayashi K, Hayashi H, Nakao F, Hayashi F: Effect of cataract surgery on intraocular pressure control in glaucoma patients. J Cataract Refract Surg 2001, 27(11):1779-1786.

28.Di Staso S, Sabetti L, Taverniti L, Aiello A, Giuffre I, Balestrazzi E: Phacoemulsification and intraocular lens implant in eyes with primary angle-closure glaucoma: our experience. Acta Ophthalmol Scand Suppl 2002, 236:17-18.

\section{Tables}

Table 1. Demographic characteristics.

\begin{tabular}{ll}
\hline & $\mathrm{n}(\%)$ or mean \pm SD (range) \\
\hline Total Eyes & 24 \\
Age, years & $64.6 \pm 11.0(44$ to 80$)$ \\
\hline Male & $14(58.3 \%)$ \\
\hline Right eyes & $14(58.3 \%)$ \\
\hline Follow up, months & $21.4 \pm 7.3(12$ to 39$)$ \\
\hline Glaucoma diagnosis & \\
\hline$\quad$ PACG & $21(87.5 \%)$ \\
\hline NTG & $3(12.5 \%)$ \\
\hline Combined with GSL & $19(79.2 \%)$ \\
\hline Preoperative medication & $1.1 \pm 0.9(0$ to 3$)$ \\
\hline Preoperative IOP, mmHg & $13.8 \pm 3.3(8.1-17.9)$ \\
\hline Baseline VA, logMAR & $0.93 \pm 0.73(0.1$ to 2.6$)$ \\
\hline Baseline BCVA, logMAR & $0.59 \pm 0.67(0$ to 2.6$)$ \\
\hline Vertical cup disc ratio & $0.95 \pm 0.09(0.7$ to 1$)$ \\
\hline Visual field & \\
\hline Stage 4 & $19(79.2 \%)$ \\
\hline Stage 5 & $5(20.8 \%)$ \\
\hline MD, dB & $-29.16 \pm 4.20(-21.4$ to $-33 \square$ \\
\hline VFI, \% & $13.38 \pm 11.46(0-37)$ \\
\hline
\end{tabular}


$\mathrm{SD}=$ standard deviation; PACG = primary angle closure glaucoma; NTG = normal tension glaucoma; GSL = goniosynechialysis; IOP = intraocular pressure; VA = visual acuity; $\log \mathrm{MAR}=$ logarithm of the minimum angle of resolution; $\mathrm{BCVA}=$ best corrected visual acuity; $\mathrm{MD}=$ mean deviation, $\mathrm{dB}=$ decibel, $\mathrm{VFI}=$ visual field index.

Table 2. VA changes in postoperative visits

\begin{tabular}{llll}
\hline & Improved, $\mathrm{n}(\%)$ & Unchanged, $\mathrm{n}(\%)$ & Worsening, $\mathrm{n}(\%)$ \\
\hline 1-month & $20(83.3 \%)$ & $2(8.3 \%)$ & $2(8.3 \%)$ \\
6-month & $16(66.7 \%)$ & $6(25.0 \%)$ & $2(8.3 \%)$ \\
\hline Final & $14(58.3 \%)$ & $5(20.8 \%)$ & $5(20.8 \%)$ \\
\hline
\end{tabular}

$\mathrm{VA}=$ visual acuity

Table 3. Postoperative complications

\begin{tabular}{lccc} 
Complications / Groups & $\begin{array}{c}\text { Improved } \\
(\mathrm{n}=14)\end{array}$ & $\begin{array}{c}\text { Unchanged } \\
(\mathrm{n}=5)\end{array}$ & $\begin{array}{c}\text { Worsening } \\
(\mathrm{n}=5)\end{array}$ \\
\hline Posterior capsular opacity & $3(21.4 \%)$ & & \\
Malignant glaucoma & & $1(20 \%)$ & \\
Shallow anterior chamber & & $1(20 \%)$ & \\
Ischemic optic neuropathy & & $1(20 \%)$ \\
\hline
\end{tabular}

\section{Figures}

109 Glaucoma patients with cataract surgery from the high risk surgery bank (March 2015-April 2018)

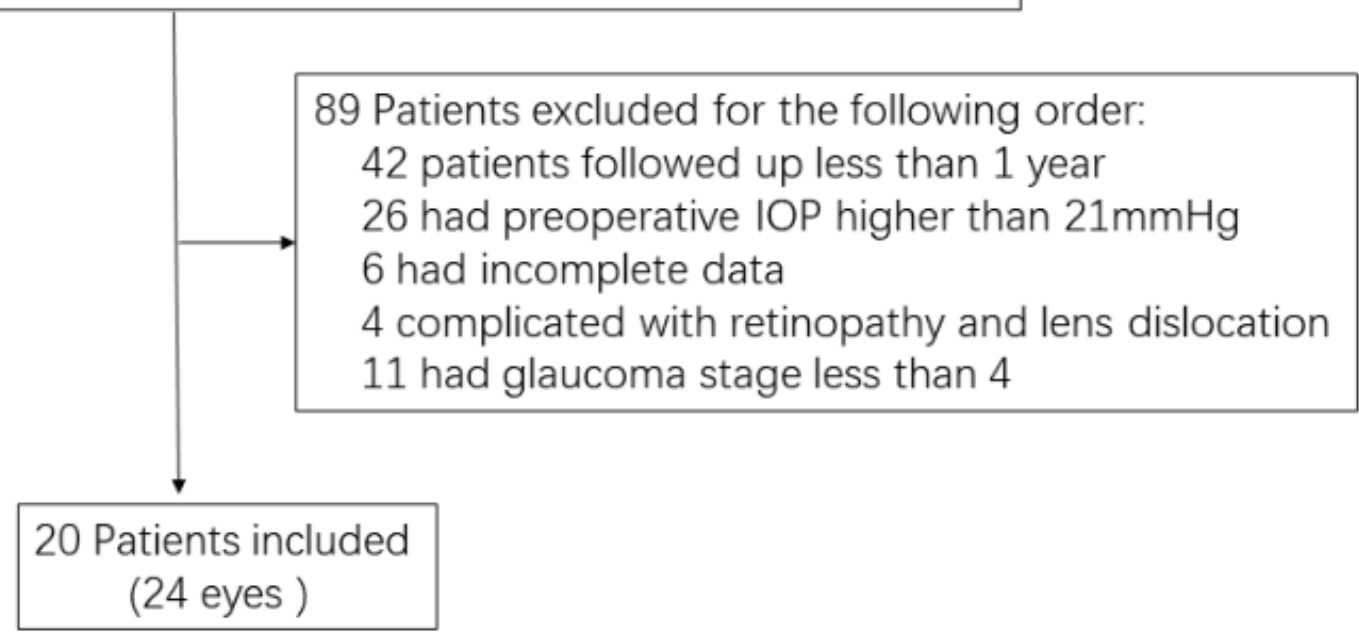


Figure 1

Flow chart of patients included in the severe and end stage glaucoma for final analysis. Total 20 patients with 24 eyes were included.

\section{Baseline 1-month 6-month Final}

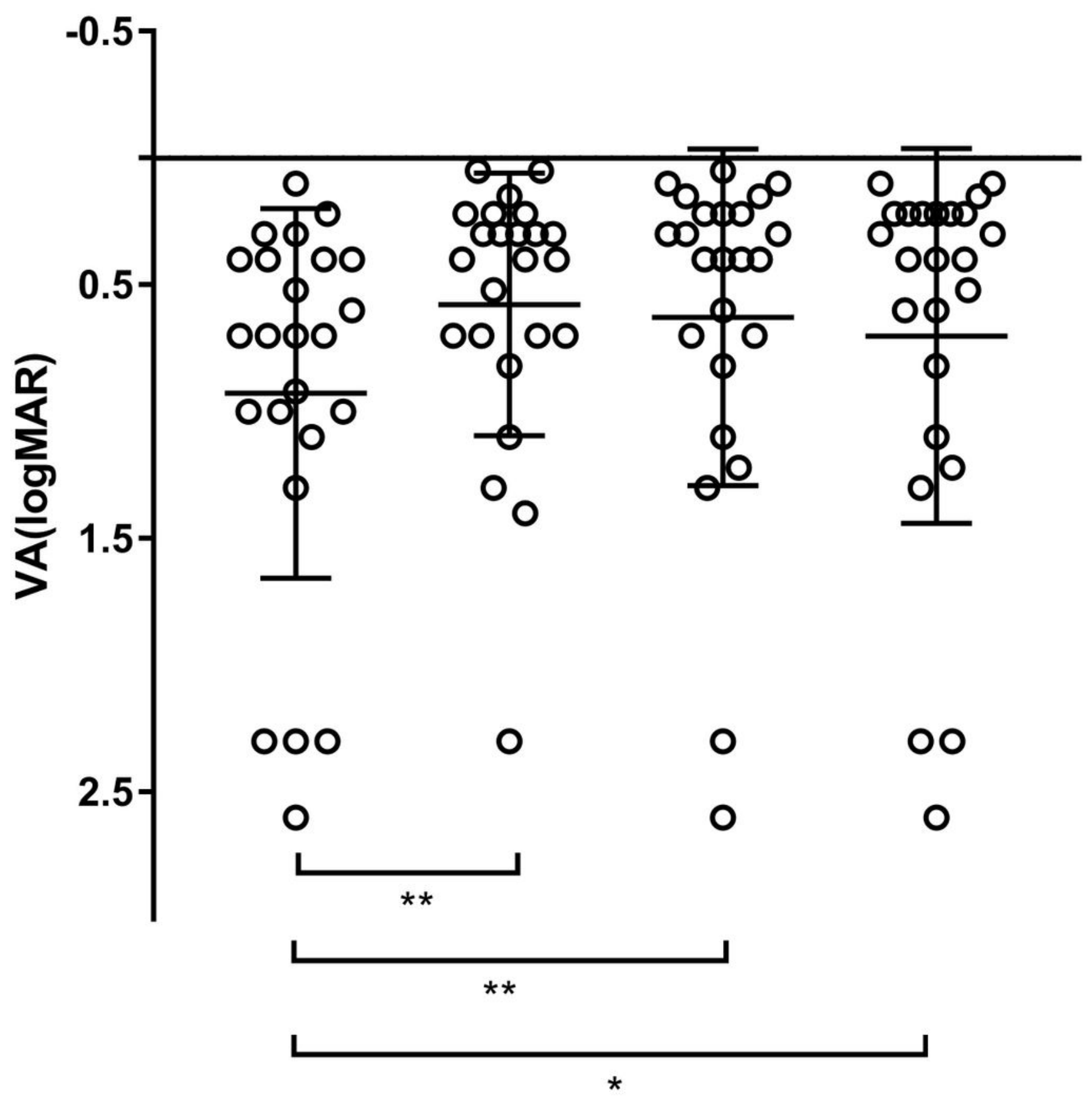

Figure 2 
Changes in preoperative visual acuity (VA) and postoperative VA. Postoperative VA levels were all significantly improved compared with preoperative VA at 1 month, 6 months and final visit. ${ }^{*} p<0.05$ and $\star * p<0.01$. $\log M A R=$ logarithm of the minimum angle of resolution. The patient number was 24 in all the visits.
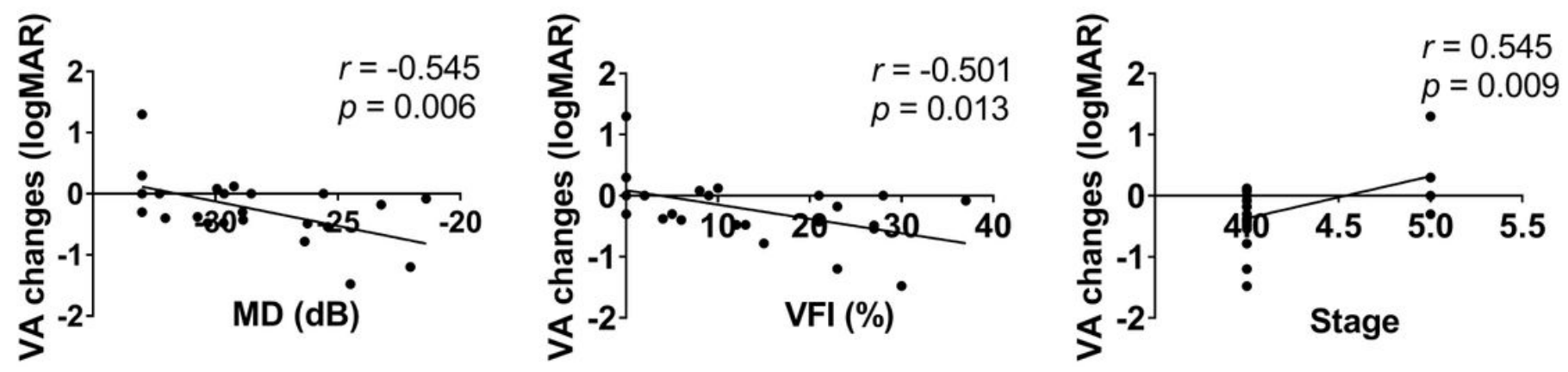

Figure 3

Scatter plots showing factors related to postoperative visual acuity (VA) changes. logMAR = logarithm of the minimum angle of resolution; $\mathrm{MD}=$ mean deviation, $\mathrm{dB}=$ decibel, $\mathrm{VFI}=$ visual field index.

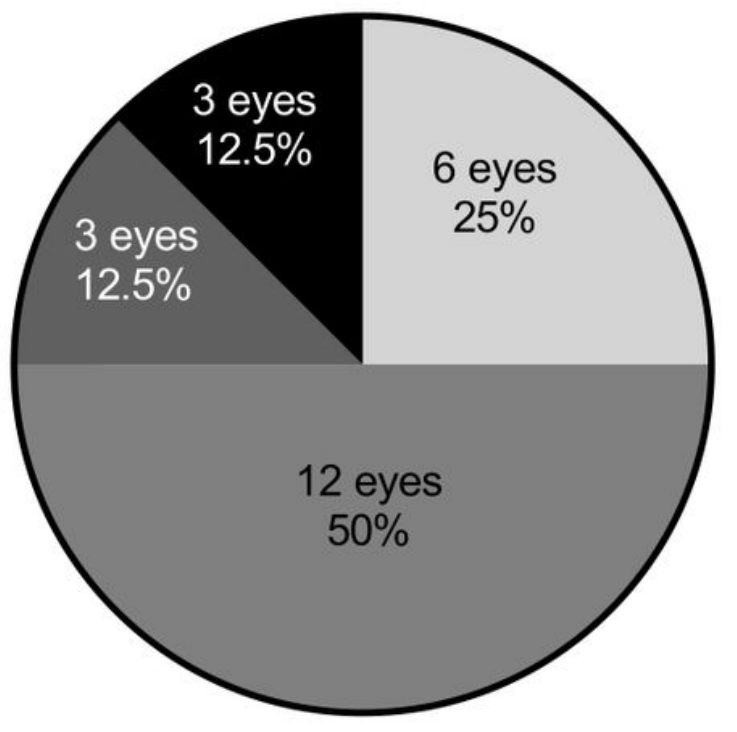

Baseline Medicaitons
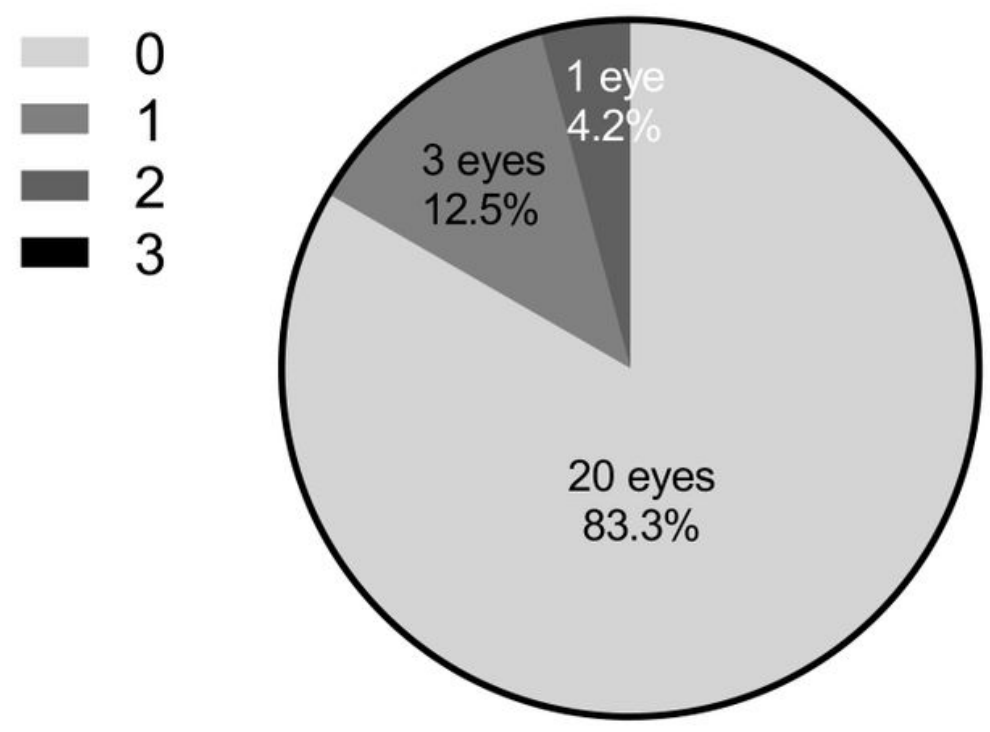

Final Medicaitons

\section{Figure 4}

The baseline and final number of medications. 